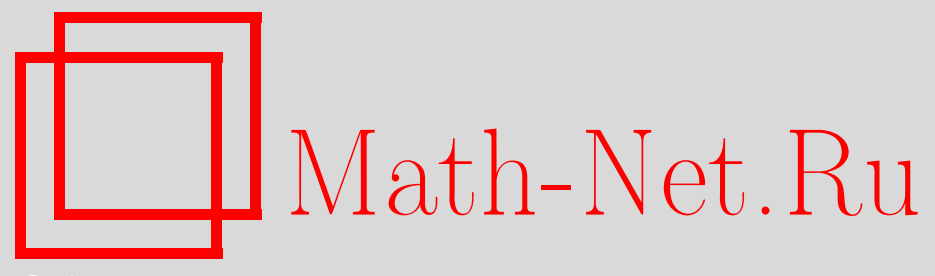

Т. П. Романова, Предельный анализ и оптимальное опирание трехслойных армированных круглых пластин из разносопротивляющихся материалов при неравномерном нагружении, Вестн. Сам. гос. техн. ун-та. Сер. Физ.-мат. науки, 2016, номер 3, 508-523

DOI: https://doi.org/10.14498/vsgtu1467

Использование Общероссийского математического портала MathNet.Ru подразумевает, что вы прочитали и согласны с пользовательским соглашением

http://www . mathnet.ru/rus/agreement

Параметры загрузки:

IP: 35.174 .16 .151

26 апреля 2023 г., 16:36:23

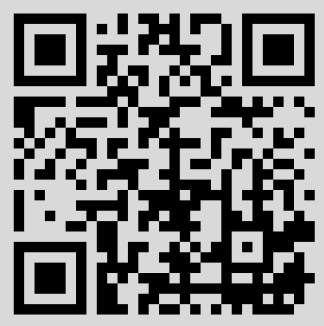


УДК 539.37:539.411.5

\title{
ПРЕДЕЛЬНЫЙ АНАЛИЗ И ОПТИМАЛЬНОЕ ОПИРАНИЕ ТРЕХСЛОЙНЫХ АРМИРОВАННЫХ КРУГЛЫХ ПЛАСТИН ИЗ РАЗНОСОПРОТИВЛЯЮЩИХСЯ МАТЕРИАЛОВ ПРИ НЕРАВНОМЕРНОМ НАГРУЖЕНИИ
}

\section{T. П. Романова}

Институт теоретической и прикладной механики им. С. А. Христиановича СО РАН, Россия, 630090, Новосибирск, ул. Институтская, 4/1.

\begin{abstract}
Аннотация
В рамках модели идеального жесткопластического тела рассмотрено предельное поведение гибридных композитных круглых пластин. Построено точное решение задачи изгиба трехслойных круглых пластин, имеющих разную структуру углового армирования в верхнем и нижнем слое. Материал среднего слоя и связующего в верхнем и нижнем слоях имеет пределы текучести на сжатие намного большие, чем на растяжение. В этом случае условие пластичности в плоскости главных моментов, построенное на основе структурной модели армированного слоя с одномерным напряженным состоянием в волокнах, имеет вид прямоугольника. Пластины шарнирно оперты по внутреннему круговому контуру и имеют в центральной части жесткую круглую вставку. Пластины находятся под действием осесимметричной поверхностной нагрузки. Показано, что в зависимости от расположения опорного контура, структуры армирования, распределения приложенной нагрузки возможны несколько схем предельного деформирования пластин; определены условия их реализации. Найдены поля главных моментов и скорости прогибов пластины. Получены простые аналитические выражения для предельной нагрузки. Определено оптимальное расположение опоры, при котором пластина имеет наименьшую повреждаемость с точки зрения максимума предельной нагрузки. Показано, что на оптимальной опоре образуется пластический шарнир. Получено, что при увеличении приложенной распределенной нагрузки в несколько раз значения предельных нагрузок уменьшатся во столько же раз, при этом месторасположение оптимальной опоры не изменится. Полученные решения могут быть использованы для оценки несущей способности трехслойных железобетонных пластин.
\end{abstract}

Ключевые слова: жесткопластическая модель, гибридное армирование, разносопротивляющиеся материалы, угловое армирование, трехслойная пластина, неравномерная нагрузка, внутренняя опора, предельная нагрузка, оптимальная опора.

(C) 2016 Самарский государственный технический университет.

\section{Образец для цитирования}

Р ом ан о в а Т. П. Предельный анализ и оптимальное опирание трехслойных армированных круглых пластин из разносопротивляющихся материалов при неравномерном нагружении // Вестн. Сам. гос. техн. ун-та. Сер. Физ.-мат. науки, 2016. Т. 20, № 3. С. 508-523. doi: $10.14498 /$ vsgtu1467.

\section{Сведения об авторе}

Татьяна Павловна Романова (к.ф.-м.н.; lab4nemir@gmail.com), старший научный сотрудник, лаб. физики быстропротекающих процессов. 
Введение. Для изготовления разнообразных элементов конструкций, которые могут быть как однородными, так и композитными, широко используются анизотропные разносопротивляющиеся материалы. K таким элементам относятся и круглые многослойные армированные пластины. Зачастую в элементах конструкций предоставляется свобода выбора планировочных решений, в том числе и свобода размещения опор [1]. В работе [2] на примере неравномерно нагруженных железобетонных балок показано, что если условия проектирования допускают выбор расположения опорного контура элементов конструкций и если стоимость опоры не зависит от ее положения, то за счет рационального размещения опор можно получить эффект больший, чем от других способов оптимизации. С целью определения оптимального расположения контура в настоящей работе в рамках жесткопластической модели рассмотрено предельное деформирование круглых трехслойных армированных пластин из разносопротивляющихся материалов фаз композиции под действием неравномерной нагрузки, в общем случае при различных характеристиках углового армирования в несущих слоях и различном расположении опорного кругового контура внутри области пластины. В литературе точные решения для пластических армированных пластин, опертых по внутреннему контуру, под действием неравномерной нагрузки неизвестны. При нагрузке, равномерно распределенной по поверхности, аналогичная задача по определению несущей способности рассмотрена в [3]. В случае однородного материала в [4] методом предельного равновесия определена предельная нагрузка для круглой пластины, опертой на $n$ точек, расположенных симметрично на окружности. В [5] найдено оптимальное положение дополнительной внутренней опоры для шарнирно опертой, защемленной и свободной на контуре круглой пластины под действием начального импульса. В $[6,7]$ методом предельного равновесия для квадратных и прямоугольных плит определено оптимальное размещение колонн, рассматриваемых как точечные опоры. Изгиб и вопросы оптимального выбора опор для однородных упругих, упругопластических круглых, прямоугольных и полосовых пластин с промежуточными опорами изучены в [8-11]. Оптимальное положение полигональных внутренних опор к однородным жесткопластическим круглым, одно- и двусвязным полигональным пластинам найдено в [12,13] методом предельного равновесия. Динамическое поведение однородных криволинейных двусвязных пластин с внутренней опорой рассмотрено в [14]. Проблемы оптимального проектирования строительных конструкций с точки зрения перспектив использования найденных решений при разработке реальных объектов массового строительства обсуждаются в [15].

1. Условие пластичности и закон пластического течения. Рассмотрим в полярных координатах $(r, \varphi)$ круглую тонкую кирхгофовскую пластину с центральной круглой жесткой вставкой с контуром $L_{0}$, шарнирно опертую по внутреннему круговому контуру $L_{1}$, при воздействии неравномерно распределенной нагрузки, зависящей от радиуса $r$. Внешний контур пластины $L_{2}$ свободный (рис. 1, 2; значения безразмерных параметров на рисунках приведены ниже). Окружности $L_{0}, L_{1}$ и $L_{2}$ являются концентрическими; $R_{0}, R_{1}$ и $R_{2}$ - их радиусы $\left(0 \leqslant R_{0} \leqslant R_{1} \leqslant R_{2}\right)$. Как и в [3], пластина состоит из трех различных слоев. Верхний $I_{1}$ и нижний $I_{2}$ слои (несущие слои) содержат по толщине большое количество армированных слоев и связующих их изо- 


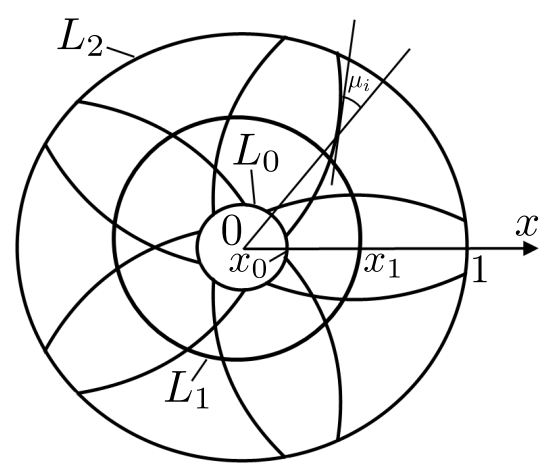

Рис. 1. Круглая пластина с угловым армированием

[Figure 1. A circular plate with corner reinforcement]

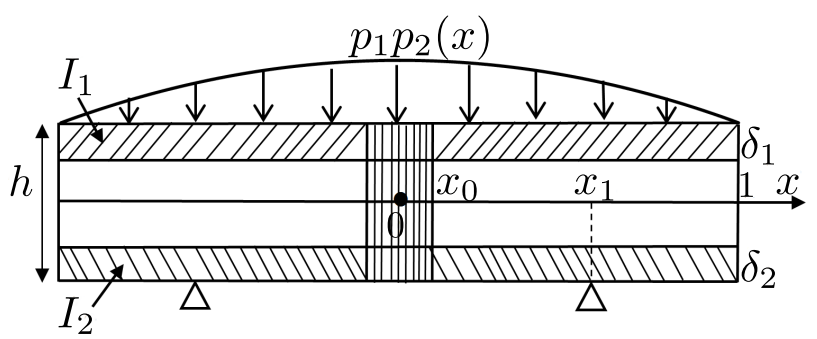

Рис. 2. Круглая пластина в поперечном сечении

[Figure 2. A circular plate in the cross section]

тропных прослоек, описываемых моделью идеального жесткопластического материала с условием пластичности типа модифицированного условия Треска для материала, разносопротивляющегося на растяжение и сжатие. Считается, что волокна арматуры деформируются, как одномерные элементы, располагаются в виде симметричных относительно радиуса криволинейных траекторий (угловое армирование), армирование в верхнем и нижнем слое различное. Средний слой пластины выполнен из того же материала, что связующий материал в несущих слоях.

Пусть $\bar{M}_{1}, \bar{M}_{2}$ - радиальный и окружной изгибные моменты; $m_{1}$ и $m_{2}-$ их безразмерные величины; $k \sigma_{0}$ и $\sigma_{0}$ - пределы текучести связующего материала на растяжение и сжатие $(0<k \leqslant 1), s_{i}^{a}$ - предел текучести материала арматуры в слое $I_{i}(i=1,2) ; \mu_{i}$ - угол армирования угловых волокон в области $I_{i} ; \omega_{i}(x), \omega_{i 0}$ - плотность армирования волокон в области $I_{i}$ и ее значение при $x=x_{0} ; \bar{\delta}_{1}$ и $\bar{\delta}_{2}$ - толщины верхнего и нижнего слоев; $\bar{w}-$ прогиб; $\bar{t}-$ время; $H$ - толщина пластины; $\kappa_{1}, \kappa_{2}$ - главные скорости кривизны поверхности пластины (безразмерные); $\sigma_{0}^{0}, H_{0}, t_{0}$ - параметры обезразмеривания:

$$
\begin{gathered}
s=\sigma_{0} / \sigma_{0}^{0}, \quad m_{i}=\bar{M}_{i} / M_{0}^{0}, \quad M_{0}^{0}=\sigma_{0}^{0} H_{0}^{2} / 4, \quad s_{i}=s_{i}^{a} / \sigma_{0}^{0}, \quad \delta_{i}=\bar{\delta}_{i} / H_{0} ; \\
\omega_{i 1}=2 \omega_{i} \cos ^{2} \mu_{i}, \quad \omega_{i 2}=2 \omega_{i} \sin ^{2} \mu_{i}, \quad \mu_{i 0}=\mu_{i}\left(x_{0}\right) \quad(i=1,2) ; \\
h=H / H_{0}, \quad \kappa_{1}=-\dot{v}^{\prime \prime}, \quad \kappa_{2}=-\dot{v}^{\prime} / x, \quad x=r / R_{2}, \quad x_{j}=R_{j} / R_{2} \quad(j=0,1) ; \\
v=\left(H_{0} / R_{2}\right)^{2} w, \quad w=\bar{w} / H_{0}, \quad(\cdot)^{\prime}=\partial(\cdot) / \partial x, \quad(\cdot)=\partial(\cdot) / \partial t, \quad t=\bar{t} / t_{0} .
\end{gathered}
$$


Для возможных вариантов структур углового армирования плотность армирования одного семейства волокон $\omega_{i}(x)(i=1,2)$ определяется как в [16]:

а) спирали Архимеда:

$$
\omega_{i}(x)=\frac{\omega_{i 0} \sqrt{x_{0}^{2}+\left(x \operatorname{tg} \mu_{i}\right)^{2}}}{x \sqrt{1+\operatorname{tg}^{2} \mu_{i}}}, \quad \operatorname{tg} \mu_{i}=\frac{x}{x_{0}} \operatorname{tg} \mu_{i 0} ;
$$

б) логарифмические спирали:

$$
\omega_{i}(x)=\frac{\omega_{i 0} x_{0}}{x}, \quad \mu_{i}(x)=\mu_{i 0}=\mathrm{const}
$$

в) «спицы велоколеса»:

$$
\omega_{i}(x)=\frac{\omega_{i 0} x_{0} \cos \mu_{i 0}}{\sqrt{x^{2}-\left(x_{0} \sin \mu_{i 0}\right)^{2}}}, \quad \sin \mu_{i}=\frac{x_{0}}{x} \sin \mu_{i 0} .
$$

В работе [3] показано, что в случае, когда отношение пределов текучести связующего материала на растяжение и сжатие $k$ мало (например, это справедливо для бетона в случае железобетонных пластин), условие пластичности, полученное на основе структурной модели армированного слоя с одномерным напряженным состоянием в волокнах $[17,18]$ имеет вид прямоугольника $A B C D$, изображенного на рис. 3:

- режим $A B: m_{2}=a_{2}(x), \kappa_{1}=0, \kappa_{2}>0$;

- режим $B C: m_{1}=-a_{3}(x), \kappa_{2}=0, \kappa_{1}<0$;

- режим $C D: m_{2}=-a_{4}(x), \kappa_{1}=0, \kappa_{2}<0$;

- pежим $A D: m_{1}=a_{1}(x), \kappa_{2}=0, \kappa_{1}>0$,

где

$$
\begin{gathered}
a_{1}=2\left(k s h^{2}+\delta_{1}^{2}\left(2 s \omega_{1}-s_{1} \omega_{11}\right)-\delta_{2}\left(2 h-\delta_{2}\right)\left(2 k s \omega_{2}-s_{2} \omega_{21}\right)-\right. \\
\left.-\frac{\left(k s h+\delta_{1}\left(2 s \omega_{1}-s_{1} \omega_{11}\right)-\delta_{2}\left(2 k s \omega_{2}-s_{2} \omega_{21}\right)\right)^{2}}{(k+1) s}\right), \\
a_{2}=2\left(k s h^{2}+\delta_{1}^{2}\left(2 s \omega_{1}-s_{1} \omega_{12}\right)-\delta_{2}\left(2 h-\delta_{2}\right)\left(2 k s \omega_{2}-s_{2} \omega_{22}\right)-\right. \\
\left.-\frac{\left(k s h+\delta_{1}\left(2 s \omega_{1}-s_{1} \omega_{12}\right)-\delta_{2}\left(2 k s \omega_{2}-s_{2} \omega_{22}\right)\right)^{2}}{(k+1) s}\right) ;
\end{gathered}
$$

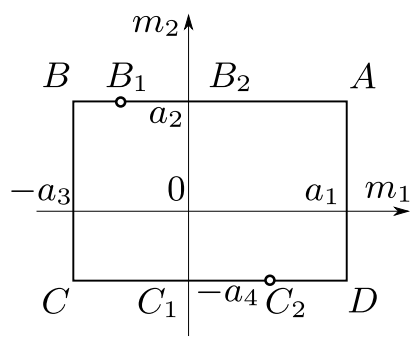

Рис. 3. Условие пластичности в безразмерных главных моментах $m_{1}, m_{2}$

[Figure 3. The plasticity condition for dimensionless principal moments $m_{1}, m_{2}$ ] 


$$
\begin{gathered}
a_{3}=2\left(s h^{2}+\delta_{1}^{2}\left(2 k s \omega_{1}-s_{1} \omega_{11}\right)-\delta_{2}\left(2 h-\delta_{2}\right)\left(2 s \omega_{2}-s_{2} \omega_{21}\right)-\right. \\
\left.-\frac{\left(s h+\delta_{1}\left(2 k s \omega_{1}-s_{1} \omega_{11}\right)-\delta_{2}\left(2 s \omega_{2}-s_{2} \omega_{21}\right)\right)^{2}}{(k+1) s}\right), \\
a_{4}=2\left(s h^{2}+\delta_{1}^{2}\left(2 k s \omega_{1}-s_{1} \omega_{12}\right)-\delta_{2}\left(2 h-\delta_{2}\right)\left(2 s \omega_{2}-s_{2} \omega_{22}\right)-\right. \\
\left.-\frac{\left(s h+\delta_{1}\left(2 k s \omega_{1}-s_{1} \omega_{12}\right)-\delta_{2}\left(2 s \omega_{2}-s_{2} \omega_{22}\right)\right)^{2}}{(k+1) s}\right) .
\end{gathered}
$$

Считаем, что $a_{j}(x)(j=1,2,3,4)$ являются гладкими функциями.

2. Определение моментов, предельной нагрузки и скоростей. Уравнения равновесия круглой пластины имеют вид

$$
\begin{aligned}
& \left(x m_{1}\right)^{\prime}-m_{2}=x q, \\
& (x q)^{\prime}=-x p_{1} p_{2}(x),
\end{aligned}
$$

где $q=\bar{Q} R_{2} / M_{0}^{0} ; p_{i}=\bar{P}_{i} R_{2}^{2} / M_{0}^{0}(i=1,2) ; \bar{Q}$ - перерезывающая сила, $\bar{P}=$ $=\bar{P}_{1} \bar{P}_{2}(r)$ - нагрузка, распределенная по поверхности пластины. Пусть опорная окружность смещена от внешнего контура пластины немного внутрь ее $\left(x_{12} \leqslant x_{1} \leqslant 1\right.$; значение $x_{12}$ определим ниже). Назовем возникающую при этом схему предельного деформирования схемой 1 . Окружность $x=x_{1}$ является промежуточным шарниром, на котором справедливо неравенство

$$
-a_{3}\left(x_{1}\right)<m_{1}\left(x_{1}\right) \leqslant 0
$$

и перерезывающие силы терпят разрыв. Считаем, что на отрезке $x_{0} \leqslant x \leqslant x_{1}$ реализуется пластическое состояние $A B_{1}$, при котором

$m_{2}(x)=a_{2}(x), \dot{v}^{\prime \prime}=0, m_{1}\left(x_{1}\right) \leqslant m_{1}(x) \leqslant a_{1}(x), m_{1}\left(x_{1}\right) \leqslant 0, m_{1}\left(x_{0}\right)=a_{1}\left(x_{0}\right)$,

а на отрезке $x_{1} \leqslant x \leqslant 1$ реализуется состояние $B_{1} B_{2}$, при котором

$$
m_{2}(x)=a_{2}(x), \quad \dot{v}^{\prime \prime}=0, \quad m_{1}\left(x_{1}\right) \leqslant m_{1}(x) \leqslant 0, \quad m_{1}\left(x_{1}\right) \leqslant 0, \quad m_{1}(1)=0 .
$$

Из уравнения $\dot{v}^{\prime \prime}=0$ при учете равенства нулю скоростей прогибов на опорном контуре $x=x_{1}$ и того, что жесткая область не деформируется, получим распределение скоростей прогибов при схеме 1 (рис. 4, a; $\dot{v}_{0}$ - безразмерная скорость прогиба жесткой шайбы):

$$
\dot{v}=\left\{\begin{array}{cc}
\dot{v}_{0} & \text { при } 0 \leqslant x \leqslant x_{0} ; \\
\dot{v}_{0} \frac{x_{1}-x}{x_{1}-x_{0}} & \text { при } x_{0} \leqslant x \leqslant 1 .
\end{array}\right.
$$

Из (7)-(9) при $x_{0} \leqslant x \leqslant x_{1}$ имеем

$$
x q(x)=x_{0} q\left(x_{0}\right)-p_{1} \int_{x_{0}}^{x} p_{2}(y) y d y ; \quad x_{0} q\left(x_{0}\right)=-p_{1} \int_{0}^{x_{0}} p_{2}(y) y d y
$$



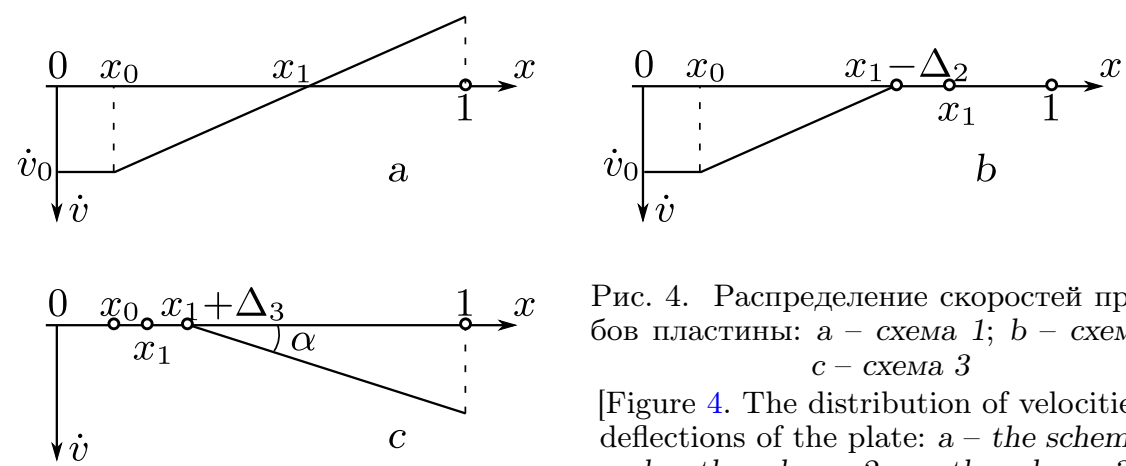

Рис. 4. Распределение скоростей прогибов пластины: a - схема $1 ; b-$ схема 2 ; $c$ - схема 3

[Figure 4. The distribution of velocities of deflections of the plate: a - the scheme 1; $b$ - the scheme $2 ; c-$ the scheme 3$]$

$$
\begin{gathered}
\left(x m_{1}\right)^{\prime}=a_{2}(x)-p_{1} \int_{0}^{x} p_{2}(y) y d y \\
m_{1}(x)=\frac{x_{0}}{x} a_{1}\left(x_{0}\right)+\frac{1}{x} \int_{x_{0}}^{x} a_{2}(y) d y-\frac{p_{1}}{x} \int_{x_{0}}^{x} \int_{0}^{\bar{x}} p_{2}(y) y d y d \bar{x} .
\end{gathered}
$$

При $x_{1} \leqslant x \leqslant 1$, учитывая $q(1)=0$, из $(7),(8),(10)$ получим

$$
\begin{gathered}
x q(x)=-p_{1} \int_{1}^{x} p_{2}(y) y d y ; \quad\left(x m_{1}\right)^{\prime}=a_{2}(x)-p_{1} \int_{1}^{x} p_{2}(y) y d y \\
m_{1}(x)=-\frac{1}{x} \int_{x}^{1} a_{2}(y) d y-\frac{p_{1}}{x} \int_{x}^{1} \int_{\bar{x}}^{1} p_{2}(y) y d y d \bar{x}
\end{gathered}
$$

Из равенства

$$
m_{1}\left(x_{1}-0\right)=m_{1}\left(x_{1}+0\right)
$$

и (11), (12) получим предельную нагрузку $p_{01}$ для схемы 1 :

$$
\begin{aligned}
p_{01}=\left(x_{0} a_{1}\left(x_{0}\right)+\int_{x_{0}}^{1} a_{2}(x) d x\right) \times & \\
& \times\left(\int_{x_{0}}^{x_{1}} \int_{0}^{\bar{x}} p_{2}(y) y d y d \bar{x}-\int_{x_{1}}^{1} \int_{\bar{x}}^{1} p_{2}(y) y d y d \bar{x}\right)^{-1} .
\end{aligned}
$$

Подставляя $p_{1}=p_{01}$ в $(11),(12)$, получим значения радиального момента $m_{1}(x)$ :

$$
\begin{aligned}
& x m_{1}(x)=x_{0} a_{1}\left(x_{0}\right)+\int_{x_{0}}^{x} a_{2}(x) d x- \\
&-\left(x_{0} a_{1}\left(x_{0}\right)+\int_{x_{0}}^{1} a_{2}(x) d x\right) \int_{x_{0}}^{x} \int_{0}^{\bar{x}} p_{2}(y) y d y d \bar{x} \times \\
& \times\left(\int_{x_{0}}^{x_{1}} \int_{0}^{\bar{x}} p_{2}(y) y d y d \bar{x}-\int_{x_{1}}^{1} \int_{\bar{x}}^{1} p_{2}(y) y d y d \bar{x}\right)^{-1} \\
& \text { при } x_{0} \leqslant x \leqslant x_{1} ;
\end{aligned}
$$




$$
\begin{aligned}
& x m_{1}(x)=- \int_{x}^{1} a_{2}(x) d x-\left(x_{0} a_{1}\left(x_{0}\right)+\int_{x_{0}}^{1} a_{2}(x) d x\right) \int_{x}^{1} \int_{\bar{x}}^{1} p_{2}(y) y d y d \bar{x} \times \\
& \times\left(\int_{x_{0}}^{x_{1}} \int_{0}^{\bar{x}} p_{2}(y) y d y d \bar{x}-\int_{x_{1}}^{1} \int_{\bar{x}}^{1} p_{2}(y) y d y d \bar{x}\right)^{-1} \\
& \text { при } x_{1} \leqslant x \leqslant 1 ;
\end{aligned}
$$

Из анализа (14), (15) следует, что $m_{1}(x)$ убывает при $x_{0} \leqslant x \leqslant x_{1}$, возрастает при $x_{1} \leqslant x \leqslant 1$ и достигает минимального значения при $x=x_{1}$. При условии

$$
m_{1}\left(x_{1}\right)=-a_{3}\left(x_{1}\right)
$$

на опоре образуется пластический шарнир. Отсюда следует, что схема 1 реализуется для значений $x_{1}$ в интервале $x_{12} \leqslant x_{1} \leqslant 1$, где $x_{12}$ определяется из уравнения

$$
m_{1}\left(x_{12}\right)=-a_{3}\left(x_{12}\right),
$$

которое с учетом (14), (15) имеет вид

$$
\begin{aligned}
& a_{3}\left(x_{12}\right) x_{12}=\int_{x_{12}}^{1} a_{2}(x) d x+\left(x_{0} a_{1}\left(x_{0}\right)+\int_{x_{0}}^{1} a_{2}(x) d x\right) \int_{x_{12}}^{1} \int_{\bar{x}}^{1} p_{2}(y) y d y d \bar{x} \times \\
& \times\left(\int_{x_{0}}^{x_{12}} \int_{0}^{\bar{x}} p_{2}(y) y d y d \bar{x}-\int_{x_{12}}^{1} \int_{\bar{x}}^{1} p_{2}(y) y d y d \bar{x}\right)^{-1} \cdot
\end{aligned}
$$

При радиусе опорного контура $x_{1} \leqslant x_{12}$ на контурах $L_{1 k}\left(k=2,3, L_{1 k}-\right.$ окружность радиуса $\left.x_{1}-(-1)^{k} \Delta_{k}, \Delta_{k} \geqslant 0\right)$ образуется пластический шарнир, при этом пластина деформируется как две независимые области: внутри контура $L_{12}$ (схема 2$)$ и между контурами $L_{13}$ и $L_{2}$ (схема 3$)$.

При схеме 2 будет деформироваться только часть пластины внутри контура $L_{12}$ как пластина с жесткой шайбой, защемленная по контуру $L_{12}$. На отрезке $x_{0} \leqslant x \leqslant x_{1}-\Delta_{2}\left(x_{1} \leqslant x_{12}\right)$ реализуется пластическое состояние $A B$, при котором

$$
\begin{aligned}
& m_{2}(x)=a_{2}(x), \quad \dot{v}^{\prime \prime}=0, \quad-a_{3}(x) \leqslant m_{1}(x) \leqslant a_{1}(x), \\
& m_{1}\left(x_{1}-\Delta_{2}\right)=-a_{3}\left(x_{1}-\Delta_{2}\right), \quad m_{1}\left(x_{0}\right)=a_{1}\left(x_{0}\right) .
\end{aligned}
$$

Из условия $\dot{v}^{\prime \prime}=0$ скорости прогибов в пластине при схеме 2 определяются так (рис. $4, b)$ :

$$
\dot{v}=\left\{\begin{array}{cl}
\dot{v}_{0} & \text { при } 0 \leqslant x \leqslant x_{0} \\
\dot{v}_{0} \frac{x_{1}-\Delta_{2}-x}{x_{1}-\Delta_{2}-x_{0}} & \text { при } x_{0} \leqslant x \leqslant x_{1}-\Delta_{2} .
\end{array}\right.
$$

Радиальный момент $m_{1}(x)$ определяется выражением (11). Из условия

$$
m_{1}\left(x_{1}-\Delta_{2}\right)=-a_{3}\left(x_{1}-\Delta_{2}\right)
$$


получим предельную нагрузку $p_{02}$ для схемы 2:

$$
\begin{aligned}
p_{02}=\min _{\Delta_{2} \geqslant 0}\left\{\left(a_{3}\left(x_{1}-\Delta_{2}\right)\left(x_{1}-\Delta_{2}\right)\right.\right. & \left.+a_{1}\left(x_{0}\right) x_{0}+\int_{x_{0}}^{x_{1}-\Delta_{2}} a_{2}(x) d x\right) \times \\
& \left.\times\left(\int_{x_{0}}^{x_{1}-\Delta_{2}} \int_{0}^{\bar{x}} p_{2}(y) y d y d \bar{x}\right)^{-1}\right\} .
\end{aligned}
$$

При схеме 3 будет деформироваться только часть пластины между контурами $L_{13}$ и $L_{2}$ как пластина, защемленная по внутреннему контуру $L_{13}$ и свободная на внешнем контуре $L_{2}$. На контуре $L_{13}$ образуется пластический шарнир и

$$
m_{2}\left(x_{1}+\Delta_{3}\right)<0, \quad m_{1}\left(x_{1}+\Delta_{3}\right)<0 .
$$

При $x_{1}+\Delta_{3} \leqslant x \leqslant 1$ реализуется состояние $C C_{1}$ :

$$
\begin{gathered}
m_{2}(x)=-a_{4}(x), \quad \dot{v}^{\prime \prime}=0, \quad-a_{3}(x) \leqslant m_{1}(x) \leqslant 0, \\
m_{1}\left(x_{1}+\Delta_{3}\right)=-a_{3}\left(x_{1}+\Delta_{3}\right), \quad m_{1}(1)=0 .
\end{gathered}
$$

Из условия $\dot{v}^{\prime \prime}=0$ скорости прогибов в пластине при схеме 3 определяются так (рис. $4, c)$ :

$$
\dot{v}=\alpha\left(x-x_{1}-\Delta_{3}\right) \text { при } x_{1}+\Delta_{3} \leqslant x \leqslant 1 \quad(\alpha=\text { const }) .
$$

При $x_{1}+\Delta_{3} \leqslant x \leqslant 1$, учитывая $q(1)=0$, из $(7),(8),(18)$ получим

$$
\begin{gathered}
x q(x)=-p_{1} \int_{1}^{x} p_{2}(y) y d y ; \quad\left(x m_{1}\right)^{\prime}=-a_{4}(x)-p_{1} \int_{1}^{x} p_{2}(y) y d y \\
m_{1}(x)=-\frac{1}{x} \int_{1}^{x} a_{4}(y) d y+\frac{p_{1}}{x} \int_{1}^{x} \int_{\bar{x}}^{1} p_{2}(y) y d y d \bar{x} .
\end{gathered}
$$

Предельную нагрузку $p_{03}$ для схемы 3 определим из (19) и условия

$$
m_{1}\left(x_{1}+\Delta_{3}\right)=-a_{3}\left(x_{1}+\Delta_{3}\right):
$$

$$
\begin{aligned}
p_{03}=\min _{\Delta_{3} \geqslant 0}\left\{\left(a_{3}\left(x_{1}+\Delta_{3}\right)\left(x_{1}+\Delta_{3}\right)+\right.\right. & \left.\int_{x_{1}+\Delta_{3}}^{1} a_{4}(x) d x\right) \times \\
& \left.\times\left(\int_{x_{1}+\Delta_{3}}^{1} \int_{\bar{x}}^{1} p_{2}(y) y d y d \bar{x}\right)^{-1}\right\} .
\end{aligned}
$$

Из выражения (17) видно, что предельная нагрузка $p_{02}$ возрастает при $x_{1} \rightarrow x_{0}$. Из $(20)$ следует, что величина $p_{03}$ убывает при $x_{1} \rightarrow x_{0}$. Из равенства

$$
p_{02}\left(x_{1 m}\right)=p_{03}\left(x_{1 m}\right)
$$

вычисляется значение $x_{1}=x_{1 m}>x_{0}$, при котором схема 3 переходит в схему 2. С учетом (17), (20) и того, что $\Delta_{k}=0(k=2,3)$, при переходе схемы 2 
в схему 3 , величина $x_{1 m}$ определяется из следующего алгебраического уравнения:

$$
\begin{array}{r}
\left(a_{3}\left(x_{1 m}\right) x_{1 m}+a_{1}\left(x_{0}\right) x_{0}+\int_{x_{0}}^{x_{1 m}} a_{2}(x) d x\right)\left(\int_{x_{0}}^{x_{1 m}} \int_{0}^{\bar{x}} p_{2}(y) y d y d \bar{x}\right)^{-1}= \\
=\left(a_{3}\left(x_{1 m}\right) x_{1 m}+\int_{x_{1 m}}^{1} a_{4}(x) d x\right)\left(\int_{x_{1 m}}^{1} \int_{\bar{x}}^{1} p_{2}(y) y d y d \bar{x}\right)^{-1} .
\end{array}
$$

Аналогично вышеизложенному анализу можно показать, что схема, при которой в пластине реализуются режимы $C C_{2} \rightarrow C_{2} C_{1}$, невозможна.

Пластина будет деформироваться по схеме $i$, соответствующей минимальному значению $p_{0 i}(i=1,2,3)$. Предельная нагрузка $p_{0}$ для рассматриваемой пластины следующая:

$$
p_{0}\left(x_{1}\right)=\min \left\{p_{01}\left(x_{1}\right), p_{02}\left(x_{1}\right), p_{03}\left(x_{1}\right)\right\} .
$$

Если уравнения (16), (21) имеют решения на интервале $\left(x_{0}, 1\right)$, то при $x_{12} \leqslant x_{1} \leqslant 1$ реализуется схема 1 , при $x_{1 m} \leqslant x_{1} \leqslant x_{12}$ будет схема 2 и при $x_{0} \leqslant x_{1} \leqslant x_{1 m}$ - схема 3 . В силу того, что в рассматриваемой задаче функция нагрузки $p_{2}(x)$ является произвольной функцией и функции $a_{j}(x)(j=1,2,3,4)$ в соотношениях (3)-(6), определяющие армирование пластины, также зависят от радиальной координаты $x$, в случае, когда одно или оба уравнения $(16),(21)$ не имеют решения на интервале $\left(x_{0}, 1\right)$, при предельном деформировании некоторые рассмотренные схемы реализованы не будут, а пластина будет деформироваться только по двум или одной схеме.

3. Вычисление оптимальных опор. Оптимальной будем считать опору, при которой пластина имеет максимальную предельную нагрузку:

$$
p_{0 m}=\max _{x_{0} \leqslant x_{1} \leqslant 1} p_{0}\left(x_{1}\right) .
$$

Из (13), (17), (20) следует, что функции предельных нагрузок $p_{01}\left(x_{1}\right)$, $p_{02}\left(x_{1}\right)$ - убывающие по $x_{1}$, а функция $p_{03}\left(x_{1}\right)$ - возрастающая. Если уравнения $(16),(21)$ имеют решения на интервале $\left(x_{0}, 1\right)$ и поэтому возможны все три схемы предельного деформирования, то предельная нагрузка рассматриваемых пластин будет максимальна при переходе схемы 2 в схему 3, то есть при $x_{1}=x_{1 m}$. Таким образом, условие оптимальности опоры имеет вид

$$
p_{02}\left(x_{1 m}\right)=p_{03}\left(x_{1 m}\right),
$$

а равенство (21) является алгебраическим уравнением для вычисления радиуса оптимальной опорной окружности $x_{1}=x_{1 m}$. Кроме этого, видно, что на оптимальной внутренней опоре образуется пластический шарнир.

На рис. 5 изображена предельная нагрузка $p_{0}$, вычисленная по (13), (17), (20), (22) для рассматриваемых слоистых пластин, в зависимости от радиуса опоры $x_{1}$ для случаев разного распределения нагрузки по плоскости пластины. В расчетах использовались следующие значения параметров: $k=1 / 17$, $s=1, s_{1}=40, s_{2}=50, \mu_{10}=\pi / 6, \mu_{20}=\pi / 5, \omega_{10}=0.25, \omega_{20}=0.2, h=1$, $x_{0}=0.1, \delta_{1}=0.1, \delta_{2}=0.07 ;$ при этом считалось, что армирование обоих слоев 


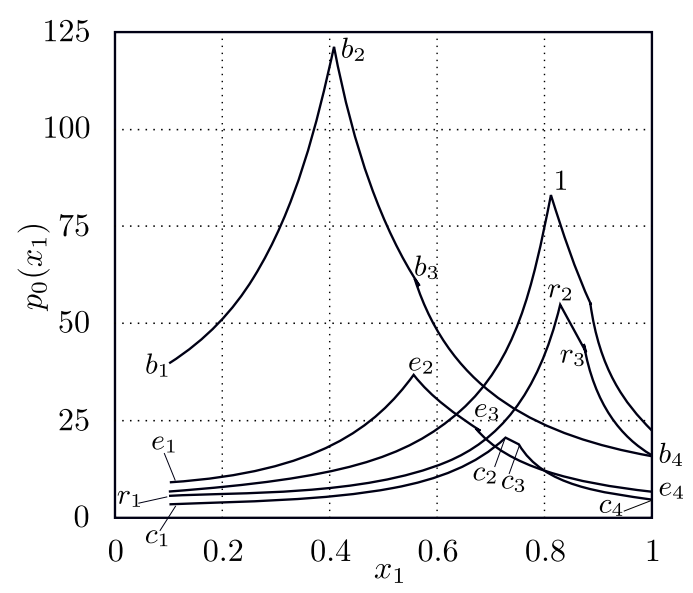

Рис. 5. Предельная нагрузка $p_{0}$ в зависимости от радиуса опоры $x_{1}$ [Figure 5. The limit load $p_{0}$ depending on the radius of the support $x_{1}$ ]

осуществлялось в форме логарифмических спиралей, описываемых законом (1).

Линия $c_{1} c_{2} c_{3} c_{4}$ изображает $p_{0}\left(x_{1}\right)$ в случае равномерной нагрузки (нагрузка 1 на рис. 6):

$$
p_{2}(x) \equiv 1
$$

На интервале $c_{1} c_{2}$ реализуется схема 3 , на интервале $c_{2} c_{3}-$ схема 2 , на интервале $c_{3} c_{4}$ - схема 1. Максимум предельной нагрузки достигается на оптимальной опоре $x_{1}=x_{1 m}=0.727$ (в точке $\left.c_{2}\right), p_{0 m}=p_{0}\left(x_{1 m}\right)=20.75$; $p_{0 m} / p_{0}(1)=4.3$.

Линия $e_{1} e_{2} e_{3} e_{4}$ изображает $p_{0}\left(x_{1}\right)$ в случае выпуклой параболической нагрузки (нагрузка 2 на рис. 6):

$$
p_{2}(x)=1-x^{2}
$$

На интервале $e_{1} e_{2}$ реализуется схема 3 , на интервале $e_{2} e_{3}-$ схема 2 , на интервале $e_{3} e_{4}-$ схема 1 . Максимум $p_{0}\left(x_{1}\right)$ достигается при $x_{1}=x_{1 m}=0.55$

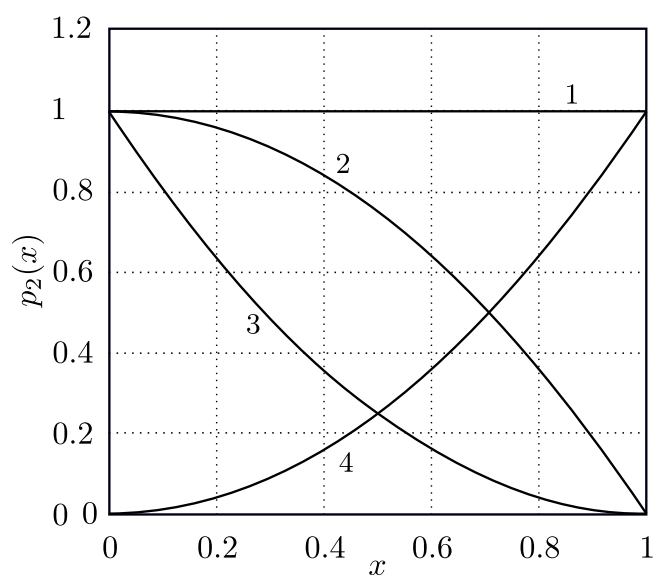

Рис. 6. Зависимости нагрузки $p_{2}$ от радиальной координаты $x$

[Figure 6. The load $p_{2}$ depending on the radial coordinate $x$ ] 
(в точке $\left.e_{2}\right), p_{0 m}=p_{0}\left(x_{1 m}\right)=36.7 ; p_{0 m} / p_{0}(1)=5.4$.

Линия $b_{1} b_{2} b_{3} b_{4}$ изображает $p_{0}\left(x_{1}\right)$ в случае вогнутой параболической нагрузки (нагрузка 3 на рис. 6):

$$
p_{2}(x)=(1-x)^{2} .
$$

На интервале $b_{1} b_{2}$ реализуется схема 3 , на интервале $b_{2} b_{3}-$ схема 2 , на интервале $b_{3} b_{4}-$ схема 1 . Максимум $p_{0}\left(x_{1}\right)$ достигается при $x_{1}=x_{1 m}=0.407$ (в точке $\left.b_{2}\right), p_{0 m}=p_{0}\left(x_{1 m}\right)=121 ; p_{0 m} / p_{0}(1)=7.56$.

Линия $r_{1} r_{2} r_{3} b_{4}$ изображает $p_{0}$ в случае вогнутой параболической нагрузки, возрастающей от центра к контуру (нагрузка 4 на рис. 6):

$$
p_{2}(x)=x^{2} \text {. }
$$

На интервале $r_{1} r_{2}$ реализуется схема 3 , на интервале $r_{2} r_{3}-$ схема 2 , на интервале $r_{3} b_{4}$ - схема 1. Максимум $p_{0}$ достигается при $x_{1}=x_{1 m}=0.83$ (в точке $\left.b_{2}\right), p_{0 m}=p_{0}\left(x_{1 m}\right)=55.2 ; p_{0 m} / p_{0}(1)=3.45$.

Если нагрузку $p_{2}(x)$ увеличить в $a_{p}$ раз, то уравнение $(21)$ для определения $x_{1 m}$ сохранит свой вид, поэтому месторасположение оптимальной опоры не изменится. При этом из (13), (17), (20), (22) следует, что значения предельных нагрузок $p_{0 i}(i=1,2,3)$ и максимальной предельной нагрузки, соответствующей оптимальному расположению опоры, уменьшатся в $a_{p}$ раз. На основании этого свойства определим величины максимальных предельных нагрузок для нагрузок вида 2-4 на рис. 6 в случае, если они имеют одинаковую с нагрузкой 1 полную распределенную нагрузку:

$$
\int_{0}^{1} p_{2}(x) x d x=\text { const }=0.5 .
$$

Тогда для нагрузок 2,4 коэффициент $a_{p}=2$, а $p_{0 m}=36.7 / 2=18.35$ и $p_{0 m}=55.2 / 2=27.6$, соответственно. Для нагрузки 3 коэффициент $a_{p} \approx 6$ и $p_{0 m}=121 / 6 \approx 20.16$.

На рис. 5 ломаная линия с максимумом в точке 1 изображает предельную нагрузку $p_{0}\left(x_{1}\right)$ для слоистых пластин, рассчитанную со следующими параметрами: $k=1 / 17, s=1, s_{1}=s_{2}=60, \mu_{10}=\pi / 6, \mu_{20}=\pi / 5, \omega_{10}=\omega_{20}=0.3$, $h=1, x_{0}=0.1, \delta_{1}=\delta_{2}=0.1$; при этом армирование обоих слоев осуществлялось в форме «спицы велоколеса», описываемой законом (2). Максимум $p_{0}$ достигается при $x_{1}=x_{1 m}=0.81, p_{0 m}=p_{0}\left(x_{1 m}\right)=83 ; p_{0 m} / p_{0}(1)=3.67$.

С помощью (13), (17) можно вычислить отношение $p_{0 m}$ к предельной нагрузке $p_{0}(1)$, когда пластина шарнирно оперта по внешнему контуру, по следующей формуле:

$$
\begin{aligned}
\frac{p_{0 m}}{p_{0}(1)}=\left(a_{3}\left(x_{1 m}\right) x_{1 m}\right. & \left.+a_{1}\left(x_{0}\right) x_{0}+\int_{x_{0}}^{x_{1 m}} a_{2}(x) d x\right) \int_{x_{0}}^{1} \int_{0}^{\bar{x}} p_{2}(y) y d y d \bar{x} \times \\
\times & {\left[\left(x_{0} a_{1}\left(x_{0}\right)+\int_{x_{0}}^{1} a_{2}(y) d y\right) \int_{x_{0}}^{x_{1 m}} \int_{0}^{\bar{x}} p_{2}(y) y d y d \bar{x}\right]^{-1}, }
\end{aligned}
$$

где $x_{1 m}$ определяется из (21). Из приведенных примеров видно, что при различных параметрах армирования и распределения нагрузки отношение $p_{0 m} / p_{0}(1)$ может быть больше семи. 
Рассмотренные примеры показывают, что изменение характеристик армирования пластины и распределения нагрузки существенно влияет на ее несущую способность. Однако если есть возможность управлять размещением опорного контура, то эффект оптимизации превосходит эффекты, получаемые за счет управления указанными параметрами. Изменяя расположение опорного контура внутри области пластины, можно найти опору, при которой пластина будет наиболее прочной.

Заключение. На основе модели идеального жесткопластического материала построено точное решение задачи по определению главных моментов, скоростей деформаций и предельной нагрузки при изгибе трехслойных круглых пластин, имеющих разную структуру углового армирования в верхнем и нижнем слое. Пластины шарнирно оперты по внутреннему круговому контуру, имеют в центральной части жесткую круглую вставку и находятся под действием неравномерно распределенной поверхностной нагрузки. Условие пластичности в плоскости главных моментов принято в виде прямоугольника, полученного на основе структурной модели армированного слоя с одномерным напряженным состоянием в волокнах с учетом разносопротивляемости материалов фаз композиции. Показано, что в зависимости от расположения опоры пластины могут деформироваться по нескольким схемам. Для всех схем получены условия их реализации, определены поля главных моментов и скорости деформаций. Получены простые аналитические выражения для предельной нагрузки в зависимости от расположения опоры. Получены алгебраические уравнения, которые определяют оптимальное расположение опорного контура, соответствующее наибольшему значению предельной нагрузки пластины и, следовательно, наименьшей ее повреждаемости при различном армировании. Получено, что на оптимальной внутренней опоре образуется пластический шарнир. Результаты проведенного предельного анализа представлены в простом аналитическом виде, удобном для дальнейшего использования. В качестве примеров рассмотрены несколько типов осесимметричных нагрузок. Показано, что изменение характеристик углового армирования, распределение нагрузки и расположение внутреннего опорного контура существенно влияет на несущую способность пластины. Получено, что при увеличении приложенной распределенной нагрузки в несколько раз значения предельных нагрузок уменьшатся во столько же раз, при этом месторасположение оптимальной опоры не изменится.

Показано, что в случае неравномерного нагружения плоских круглых элементов конструкций, если условия проектирования допускают выбор расположения опорного контура элементов конструкций и если стоимость опоры не зависит от ее положения, то за счет рационального размещения опор можно получить эффект больший, чем от других способов оптимизации. Полученные решения могут быть использованы для оценки несущей способности трехслойных железобетонных пластин.

Декларация о финансовых и других взаимоотношениях. Работа выполнена при поддержке Российского фонда фундаментальных исследований (проект № 14-01-00102-а). Автор несет полную ответственность за предоставление окончательной версии рукописи в печать. Окончательная версия рукописи была одобрена автором. Автор не получал гонорар за статью.

\section{ORCID}

Татьяна Павловна Романова: http://orcid.org/0000-0002-8563-1076 


\section{БИБЛИОГРАФИЧЕСКИЙ СПИСОК}

1. Дехтярь А. С. Точечное опирание пластин сложного очертания // Строителъная механика и расчет сооружений, 2010. № 2. С. 56-59.

2. Дехтярь А. С. Нерегулярные конструкции и целесообразность унификации // Cmpouтельная механика и расчет сооружений, 2009. № 5. С. 74-77.

3. Романова Т. П. Несущая способность и оптимизация трехслойных армированных круглых пластин из разносопротивляющихся материалов, опертых по внутреннему контуру // Проблемы прочности и пластичности, 2015. №3. C. 286-300, http://www.unn. ru/e-library/ppp.html?anum=317.

4. Yang W. H. How to optimally support a plate// J. Appl. Mech, 1981. vol. 48, no. 1. pp. 207209. doi : 10.1115/1.3157578.

5. Оленев Г. М. Оптимальное расположение дополнительных опор к жесткопластическим круглым пластинкам в случае импульсного нагружения // Уч. заn. Tapmyского гос. унma, 1983. №659. C. 30-41.

6. Дехтярь А. С. Оптимальное опирание квадратной пластины // Прикл. мех., 1991. Т. 27, № 6. C. 107-110.

7. Дехтярь А. С. Оптимальное размещение колонн в зданиях, возводимых методом подъема // Строительная механика и расчет сооружений, 1989. № 1. С. 14-17.

8. Коренева Е. Б., Гросман В. Р. Аналитическое решение задачи о неосесимметричной деформации круглой ортотропной пластины радиально-переменной толщины на точечных опорах // International Journal for Computational Civil and Structural Engineering, 2015. № 11. C. 94-100.

9. Lellep J., Polikarpus J. Optimal design of circular plates with internal supports // WSEAS Transactions on Mathematics, 2012. vol. 11, no. 3. pp. 222-232.

10. Wang C. M., Liew K. M., Wang L., Aug K. K. Optimal locations of internal line supports for rectangular plates against buckling // Structural Optimization, 1992. vol.4, no. 3. pp. 199205. doi : 10.1007/BF01742745.

11. Папковская О. Б., Козин А. Б., Камара Д. Построение и исследование решения задачи антисимметричного изгиба ортотропной полосовой пластины, подкрепленной жесткой опорой // Труды Одесского политехнического университета, 2006. № 2. С. 181-185, http://www .pratsi.opu.ua/articles/show/641.

12. Романова Т. П. Оптимальное расположение полигональных внутренних опор к круглым жесткопластическим пластинам // Вестн. Сам. гос. техн. ун-та. Сер. Физ.-мат. науки, 2014. № 3(36). С. 94-105. doi : 10.14498/vsgtu1312.

13. Романова Т. П. Оптимальное опирание жесткопластических одно- и двусвязных полигональных пластин // Вестник ПНиПУ. Механика, 2014. № 4. С. 152-177. doi: 10 . 15593/perm. mech/2014.4.06.

14. Немировский Ю. В., Романова Т. П. Моделирование поведения двусвязной жесткопластической пластины произвольной формы с внутренней криволинейной опорой при взрывных нагрузках // Проблемы прочности и пластичности, 2014. № 2. С. 122-133, http://www . unn.ru/e-library/ppp.html?anum=258.

15. Ляхович Л. С., Перельмутер А. В. Некоторые вопросы оптимального проектирования строительных конструкций// International Journal for Computational Civil and Structural Engineering, 2014. № 10(2). C. 14-23.

16. Вохмянин И. Т., Немировский Ю. В. Особенности продольно-поперечного изгиба трехслойных кольцевых пластинок с несимметричными структурами армирования / Kpaевые задачи и математическое моделирование: Сб. тр. 8-й Всерос. научн. конф-ции. Т. 1 (1-3 декабря 2006 г.). Новокузнецк, 2006. С. 25-31.

17. Немировский Ю. В., Романова Т. П. Расчет динамического деформирования трехслойных железобетонных круглых и кольцевых пластин // Бетон и железобетон, 2011. № 6 . C. $26-30$. 
18. Nemirovsky Ju. V., Resnikoff B. S. On limit equilibrium of reinforced slabs and effectiveness of their reinforcement// Archiwum Inżynierii Ladowej, 1975. vol. 21, no. 1. pp. 57-67.

Поступила в редакцию $12 / \mathrm{I} / 2016$;

в окончательном варианте - 25/VI/2016;

принята в печать - 09/IX/2016.

Vestn. Samar. Gos. Techn. Un-ta. Ser. Fiz.-mat. nauki

[J. Samara State Tech. Univ., Ser. Phys. \& Math. Sci.], 2016, vol. 20, no. 3, pp. 508-523

ISSN: 2310-7081 (online), 1991-8615 (print)

doi: http://dx.doi.org/10.14498/vsgtu1467

MSC: 74P99, 74K20, 74C99

\title{
LIMIT ANALYSIS AND OPTIMAL SUPPORT OF REINFORCED THREE-LAYER CIRCULAR PLATES OF DIFFERENT RESISTANT MATERIALS UNDER NON-UNIFORM LOADING
}

\section{T. P. Romanova}

Khristianovich Institute of Theoretical and Applied Mechanics, Siberian Branch of the Russian Academy of Sciences,

4/1, Institutskaya st., Novosibirsk, 630090, Russian Federation.

\begin{abstract}
Within the model of an ideal rigid-plastic body the limit behavior of the hybrid composite circular plates is considered. The exact solution of the problem of bending is built for three-layer reinforced circular plates having different angular structure reinforcement at the top and bottom layer. The material of the middle layer and the binder in the upper and lower layers has a yield stress in compression much greater than in tension. In this case the condition of plasticity for the main moments that are based on the structural model of the reinforced layer with one-dimensional states of stress in the fibers has the form of a rectangle of type Johansen condition. The plates are hinge supported along the internal annular contour and have the rigid circular insert in the central part. The plates are under load non-uniformly distributed over the surface of the plate. It is shown that there are a few schemes of limit deformation of the plate, depending on the location of the internal support and on distribution of load. The conditions of implementation are defined for all schemes. The main moments and the velocities of the deflections of the plate are defined at different locations of the internal support. The simple analytic expressions are obtained for the limit load. The optimal location of support is determined. The optimal support is such support, at which the plate has a maximum limit load. It is shown that the optimal position of the support corresponds to the formation of plastic
\end{abstract}

(C) 2016 Samara State Technical University.

Please cite this article in press as:

Rom an ova T. P. Limit analysis and optimal support of reinforced three-layer circular plates of different resistant materials under non-uniform loading, Vestn. Samar. Gos. Tekhn. Univ., Ser. Fiz.-Mat. Nauki [J. Samara State Tech. Univ., Ser. Phys. \& Math. Sci.], 2016, vol. 20, no. 3, pp. 508-523. doi: 10.14498/vsgtu1467. (In Russian)

Author Details:

Tatiana P. Romanova (Cand. Phys. \& Math. Sci.; lab4nemir@gmail.com), Senior Researcher, Lab. of the Physics of Fast Processes. 
hinge on it. It is obtained that with increase in the applied distributed load in several times, the limit loads will be reduced in the same times and the optimal location of the support will not change. Numerical examples are given. The solution can be useful in engineering practice to evaluate the bearing capacity of three-layer reinforced concrete plates.

Keywords: rigid-plastic model, hybrid reinforcement, different-resistant materials, corner reinforcement, three-layer plate, non-uniform loading, internal support, limit load, optimal support.

Declaration of Financial and Other Relationships. This work was supported by the Russian Foundation for Basic Research (project no. 14-01-00102-a). The author is absolutely responsible for submitting the final manuscript in print. The author has approved the final version of manuscript. The author has not received any fee for the article.

\section{ORCID}

Tatiana P. Romanova: http://orcid.org/0000-0002-8563-1076

\section{REFERENCES}

1. Dekhtyar A. S. Point supporting of plates of complicated configuration, Stroitel'naia mekhanika i raschet sooruzhenii, 2010, no. 2, pp. 56-59 (In Russian).

2. Dekhtyar A. S. Irregular structures and expedience of unification, Stroitel'naia mekhanika $i$ raschet sooruzhenii, 2009, no. 5, pp. 74-77 (In Russian).

3. Romanova T. P. Carrying capacity and optimization of three-layer reinforced circular plate of differently resistant materials, supported on the internal contour, Problems of strength and plasticity, 2015, no. 3, pp. 286-300 (In Russian).

4. Yang W. H. How to optimally support a plate, J. Appl. Mech, 1981, vol.48, no. 1, pp. 207209. doi : 10.1115/1.3157578.

5. Olenev G. M. Optimal location of additional supports to rigid-plastic circular plates in case of impulse loading, Uch. Zap. Tartu. Gos. Univ., 1983, no.659, pp. 30-41 (In Russian).

6. Dekhtyar A. S. Optimum support of a square plate, Prikl. Mekh., 1991, vol. 27, no. 6, pp. 107110 (In Russian).

7. Dekhtyar A. S. Optimal placement of columns in buildings constructed by lifting, Stroitel'naia mekhanika i raschet sooruzheni, 1989, no. 1, pp. 14-17 (In Russian).

8. Koreneva E. B, Grosman V. G. Analytical solution of unsymmetric flexure problem by circular orthotropic plate with radually varying thickness based on point supports, International Journal for Computational Civil and Structural Engineering, 2015, no. 11, pp. 94-100 (Russian).

9. Lellep J., Polikarpus J. Optimal design of circular plates with internal supports, WSEAS Transactions on Mathematics, 2012, vol. 11, no. 3, pp. 222-232.

10. Wang C. M., Liew K. M., Wang L., Aug K. K. Optimal locations of internal line supports for rectangular plates against buckling, Structural Optimization, 1992, vol. 4, no. 3, pp. 199-205. doi: $10.1007 / \mathrm{BF} 01742745$.

11. Papkovskaya O. B., Kozin O. B., Camara D. Construction and research of solution of antisymmetric flexion task for an orthotropic strip plate supported by the rigid support, Trudy Odesskogo politekhnicheskogo universiteta, 2006, no. 2, pp. 181-185 (In Russian).

12. Romanova T. P. The Optimal Location of the Polygonal Internal Supports to the Circular Rigid-Plastic Plates, Vestn. Samar. Gos. Tekhn. Univ. Ser. Fiz.-Mat. Nauki [J. Samara State Tech. Univ., Ser. Phys. \& Math. Sci.], 2014, no.3(36), pp. 94-105 (In Russian). doi: $10.14498 /$ vsgtu1312.

13. Romanova T. P. Optimal support of rigid-plastic singly and doubly connected polygonal plates, PNRPU Mechanics Bulletin, 2014, no.4, pp. 152-177 (In Russian). doi: 10.15593/ perm.mech/2014.4.06. 
14. Nemirovsky Yu. V., Romanova T. P. Modeling of behavior of doubly connected rigid-plastic plate of arbitrary form with the internal curvilinear support under explosive loads, Problems of strength and plasticity, 2014, no. 2, pp. 122-133 (In Russian).

15. Lyakhovich L. S., Perelmuter A. V. Some problems of building constructions optimal projecting, International Journal for Computational Civil and Structural Engineering, 2014, № 10(2), C. 14-23 (In Russian).

16. Vokhmianin I. T., Nemirovsky Yu. V. Features of longitudinal-transverse bending of threelayer circular plates with asymmetric structures of reinforcement, Boundary problems and mathematical modeling, Proc. of the 8th Russian Sci. Conf., vol.1. Novokuznetsk, 2006, pp. 25-31 (In Russian).

17. Nemirovsky Yu. V., Romanova T. P. Calculation of dynamic deformation of three-layer reinforced concrete circular and annular plates, Beton i Zhelezobeton, 2011, no. 6, pp. 26-30 (In Russian).

18. Nemirovsky Ju. V., Resnikoff B. S. On limit equilibrium of reinforced slabs and effectiveness of their reinforcement, Archiwum Inżynierii Ladowej, 1975, vol. 21, no. 1, pp. 57-67.

Received 12/I/2016;

received in revised form $25 / \mathrm{VI} / 2016$;

accepted 09/IX/2016. 